\title{
Identification of Electrically Stimulated Muscle after Stroke
}

\author{
Fengmin Le, Ivan Markovsky, Christopher Freeman, and Eric Rogers*
}

\begin{abstract}
The design of controllers to enable the application of Functional Electrical Stimulation as part of a rehabilitation programme for stroke patients requires an accurate model of electrically stimulated muscle. In this paper, nonlinear dynamics of the electrically stimulated muscle under isometric conditions is investigated, leading to the requirement to identify a Hammerstein model structure. Here we develop a two-stage identification method based on a preliminary construction of the linear part that is used as an initial estimate. Then the two-stage method is applied to identify the nonlinear part and optimize the linear part. The separable least squares optimization algorithm and traditional ramp deconvolution method are implemented here and compared with the proposed method using a simulated muscle system that is based on experimental data from stroke patients. The results show that the proposed method outperforms two other previously proposed methods when implemented on the simulated muscle system with different noise levels.
\end{abstract}

\section{INTRODUCTION}

Strokes affect between 174 and 216 people per 100,000 of the population in the UK each year [1] and one of the most devastating and common consequences of the stroke is loss of the use of the arm and hand [2]. Currently there is increasing interest in the application of control schemes as part of a rehabilitation programme for survivors of a stroke who are in a position to take part. Here the starting point is a research programme that has built a robotic workstation for use by stroke patients in order to regain voluntary control of their impaired arms [3]. This research is based on the application of Functional Electrical Stimulation (FES) to assist a patient's voluntary attempt to perform repeated tracking tasks using their impaired arms.

During treatment, FES is applied to the patient's triceps and Iterative Learning Control (ILC) is used to update the stimulation level in order to ensure that the assistance provided coincides as much as possible with the patient's voluntary intention. In particular, the patients hand is connected to the robot and they attempt to follow an illuminated track which represents reaching out for an object. Fig. 1 shows a stroke participant using the robotic workstation.

As they complete the task, the error between the required and achieved paths is measured and once they reach the end the robot returns their arm to the starting position, and in this resetting time an ILC algorithm is used to update the stimulation to be applied on the next attempt. This research has already had patient trials that demonstrate the basic

This work is supported by the Engineering and Physical Sciences Research Council (EPSRC). Grant no. EP/C51873X/1.

*Information: Signals, Images, Systems Research Group, School of Electronics and Computer Science, University of Southampton, Southampton, SO17 1BJ, UK

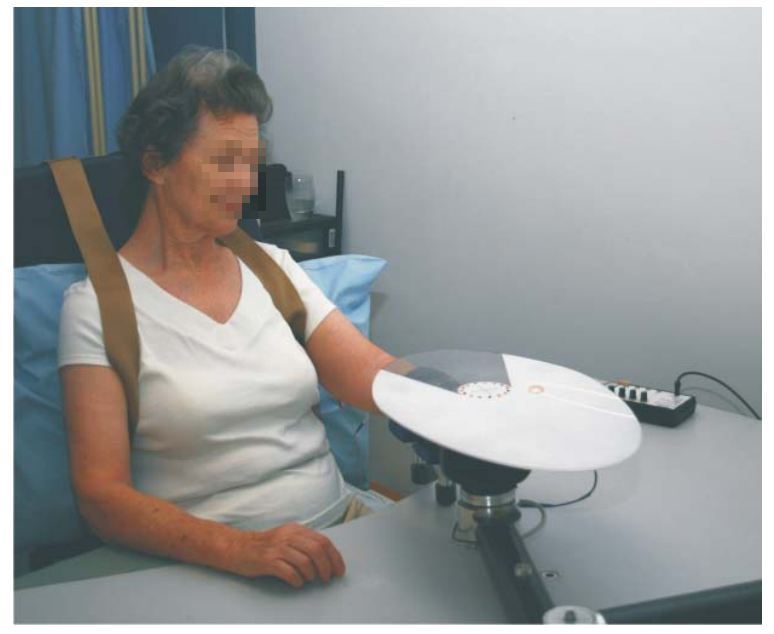

Fig. 1. The Robotic Workstation

feasibility of the approach but also highlighted the need for improved of modeling of the patient's arm and hence the motivation for the results given here.

Identification of electrically stimulated muscle is a challenging problem. The task of modeling requires both determination of a particular model structure and estimation of the model parameters that relate the model to the electrically stimulated muscle system. Although the muscular contraction mechanism is generally well known, the model based on the structure and behavior of the underlying muscle physiology [5] is still too complicated for most control applications. In order to achieve the tractability of the muscle response to electrical stimulation, attention should be focused on the accuracy of the model rather than on its transparency. To achieve this, three modeling approaches are applicable where the first is the nonlinear black box modeling approach [6]. A discrete-time NARX model is used but selection of structure, in particular model family (polynomial expansion or neural network) and the parameters for each structure need to be estimated. However, depending on the model family used, structure selection could be a difficult task. Therefore, in order to obtain an effective model, a long testing time could be needed which is highly undesirable, especially for stroke patients.

The second method is the light-grey box modeling approach where physical insights are included. In [8] and [9], three factors (activation dynamics, torque-velocity and torque-angle) are incorporated, which can be treated as coupled or not and if coupled the value of the coupled parameters are assumed to be unknown. Actually, these pa- 
rameters are approximately mutually coupled and hence they are dependent on each other. Consequently identification of these parameters in such a complex model may be practically infeasible in a reasonable experiment time period.

The third approach is a block-oriented modeling technique. One commonly used example here is the Hammerstein-Wiener model that describes nonlinear dynamic systems using one or two static nonlinear blocks in series with a linear block. In [7], a discrete-time Hammerstein model is used to describe the electrically stimulated muscle under isometric conditions (the muscle is held at a fixed length when stimulated). Later, this work was extended to non-isometric muscle [10], [14] and [13], but the key nonlinear muscle activation is still modeled by a Hammerstein structure with one or two more linear blocks in series [14][13], or parallel [10]. Using such a model to describe the muscle activation system has the possible advantages of a well defined structure and the possibility of applying a separate identification procedure for each block. Therefore, we use this modeling approach and the model structure introduced by Durfee and Palmer [10], shown in Fig. 2, is adopted.

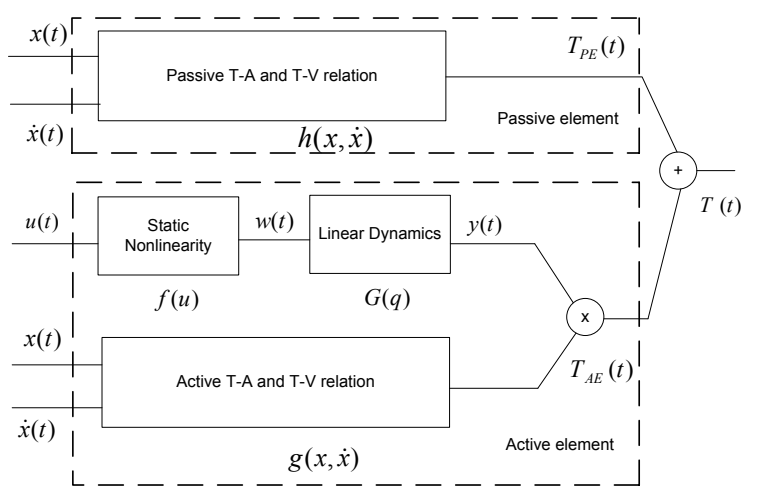

Fig. 2. Muscle Model Structure

The model input here is the stimulation pulse-width $u(t)$ $(\mu s)$, the output $T(t)$ is the torque generated at the elbow joint, $x(t)$ is the joint angle and $\dot{x}(t)$ is the joint angular velocity. The model structure consists of a passive element and an active element where the former is assumed to be time-invariant. The output of the passive element, $T_{P E}(t)$, is the torque generated by the elastic and viscous properties of the muscle without electrical stimulation. The active torque $T_{A E}(t)$ is the torque generated by the electrical stimulation of the triceps muscle and a multiplicative effect of joint angle and joint angular velocity on the resulting torque. The Passive Torque-Angle and Torque-Velocity relation $h(x, \dot{x})$ and the Active Torque-Angle and Torque-Velocity relation $g(x, \dot{x})$ represent the relationship between the joint state and the torque.

A Hammerstein structure is used to model nonlinear muscle activation in the model. It is composed of a static nonlinear block in series with linear dynamics. This is a commonly used structure due to its correspondence with biophysics. The static nonlinearity $f(u)$ includes the nerve fiber recruitment properties. This is the so called Isometric Recruitment Curve (IRC), which is defined as the static gain relation between stimulus activation level and output torque when the muscle is held at a fixed length [11]. The linear dynamic block $G(q)$ corresponds to the dynamic response of electrically stimulated muscle.

Although models of the response of electrically stimulated muscle to FES have already been identified [10], it is necessary to perform a special identification procedure with stroke patients, who exhibit different properties of torquegeneration parameters under isometric contraction [15]. The peak torque and time-dependent parameters (time to develop and reduce torque) are impaired after stroke and are independent of each other. Consequently this paper considers nonlinear muscle activation, under isometric contraction for the case of stroke patients and, in particular, the identification and relative performance of the Hammerstein structure.

Next, we develop the two-stage optimized identification procedure based on a preliminary experiment which is the main novel feature of this work. Then the separable least squares optimization algorithm [18] and traditional ramp deconvolution method [11] are implemented hand compared with the two-stage method using a simulated muscle system that is based on experimental data from stroke patient.

\section{ThE IDENTIFICATION SCHEME}

A nonlinear Hammerstein model in discrete-time is shown in Fig. 3. The stimulation input $u(\cdot)$ is first scaled by the static nonlinear function $f(\cdot)$ and then passed to a linear time-invariant system described by a transfer function $G(q)$. The system input, output and noise are denoted by $u(\cdot)$, $y(\cdot)$ and $v(\cdot)$ respectively. The internal signal $w(\cdot)$ is not measurable.

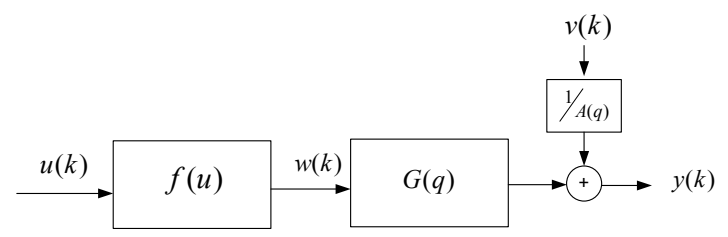

Fig. 3. Hammerstein structure

The linear system is assumed to be stable and represented by

$$
G(q)=\frac{B(q)}{A(q)}
$$

where

$$
\begin{gathered}
B(q)=b_{0} q^{-d}+b_{1} q^{-(d+1)}+\cdots+b_{n} q^{-(n+d)} \\
A(q)=1+a_{1} q^{-1}+\cdots+a_{l} q^{-l}
\end{gathered}
$$

and $B(q)$ and $A(q)$ are assumed to be coprime, $q^{-1}$ is the delay operator, and $n, l$ and $d$ are the number of zeros, poles and the time delay, respectively. These can be estimated in a number of ways where, for example, $(l, n, d)=(2,1,1)$ has been used in a related problem by [16]. 
The nonlinear function is represented by a basis function expansion $g_{p}(u)$,

$$
f(u)=\sum_{p=1}^{n} \alpha_{p} g_{p}(u)
$$

formed from scaled and translated versions of one "mother basis function" $\kappa(u)$, as

$$
g_{p}(u)=\kappa\left(\beta_{p}\left(u-\gamma_{p}\right)\right)
$$

where $\beta_{p}$ denotes the dilation parameters and $\gamma_{p}$ denotes the translation parameters [19, Pages 148-154]. A common choice is the power series

$$
g_{p}(u)=u^{p}
$$

but if, for example, deadband and saturation are present, a high order polynomial is usually required and hence the possibility of oscillatory behavior. Another method is the spline function, a piecewise polynomial function which can overcome this problem. A straightforward choice here would be a piecewise linear function, but these type of functions are not smooth, which means that their derivatives are not continuous at their break points or knots. In order to avoid these drawbacks, we will use cubic splines to model the nonlinear block. A cubic spline is a piecewise cubic function defined by a series of knot points, where between each pair of knots the spline is defined by a polynomial of order three. The second derivatives of the spline polynomials are set equal to zero at the endpoints of the interval [20, pp 49-57].

Let a set of knots $u_{1}, u_{2}, \ldots, u_{m}$ be defined which satisfy

$$
u_{\min }=u_{1}<u_{2}<u_{3}<\cdots<u_{m}=u_{\max }
$$

Then a cubic spline function is defined as

$f(u)=\sum_{i=1}^{m-2} \beta_{i}\left|u-u_{i+1}\right|^{3}+\beta_{m-1}+\beta_{m} u+\beta_{m+1} u^{2}+\beta_{m+2} u^{3}$

where $\left[\beta_{1}, \beta_{2}, \cdots, \beta_{m+2}\right]$ are parameters to be estimated for the nonlinear block and the model for the whole process is

$$
\begin{aligned}
y(k) & =G(q) f(u(k))+\frac{1}{A(q)} v(k) \\
& =\frac{B(q)}{A(q)} f(u(k))+\frac{1}{A(q)} v(k)
\end{aligned}
$$

Our task now is to estimate the parameter vector

$$
\theta=\left[a_{1}, \ldots, a_{l}, b_{0}, \ldots, b_{n}, \beta_{1}, \beta_{2}, \ldots, \beta_{m+2}\right]^{T}
$$

that minimizes the cost function

$$
V_{N}(\theta)=\frac{1}{2 N} \sum_{k=1}^{N}(y(k)-\hat{y}(k, \theta))^{2}
$$

where

$$
\hat{y}(k, \theta)=\hat{G}(q) f(u(k), \hat{\beta})
$$

from collected input output data

$$
[u(1), y(1), u(2), y(2), \ldots \ldots, u(N), y(N)]
$$

where $N$ denotes the number of samples.

\section{IDENTIFICATION PROCEDURE}

The aim of the preliminary experiment in the two-stage method proposed here is to identify the linear part as an initial estimate. The reason is that the nonlinear muscle contraction mechanism of a stroke patient is impaired and varies, see [15], and may also vary between trials. In order to obtain accurate estimates for each patient at each treatment, the identification tests need to be performed before each treatment commences. In order to avoid patient fatigue, the identification test should be as short as possible. Therefore, before each treatment, just one test is applied with the patient and then the two-stage identification procedure is used to estimate the nonlinear part and optimize the linear part.

\section{A. Preliminary experiment: PRBS test}

The Pseudo-Random Binary Signal (PRBS) changes between two levels and has white-noise-like properties. Although the PRBS signal is inappropriate for nonlinear system identification because it may not sufficiently excite the unknown nonlinearity, it is widely used in linear system identification of electrically stimulated muscle [7], [8], [16] and [17]. Therefore, PRBS input is adopted in the preliminary study and Fig. 4 shows a typical muscle response to a PRBS input.

In this application, we assume the two levels are 0 and $+c$ (where $c>0$ ) because the input pulse-width can not be negative. Also we know that $f(0)=0$ and hence we can choose $c$ such that $f(+c)=r$ (where $r \neq 0$ ) with the value of $r$ large enough compared to the possible noise level. Hence for all $k$

$$
w(k)=f(u(k))=\alpha u(k)
$$

where $\alpha=r / c$, and the output of the Hammerstein structure with PRBS inputs is

$$
\begin{aligned}
y(k) & =G(q) f(u(k))+\frac{v(k)}{A(q)} \\
& =\frac{\alpha b_{0} q^{-d}+\cdots+\alpha b_{n} q^{-(n+d)}}{1+a_{1} q^{-1}+\cdots+a_{l} q^{-l}} u(k)+\frac{1}{1+a_{1} q^{-1}+\cdots+a_{l} q^{-l}} v \\
& =\frac{\bar{b}_{0} q^{-d}+\cdots+\bar{b}_{n} q^{-(n+d)}}{1+a_{1} q^{-1}+\cdots+a_{l} q^{-l}} u(k)+\frac{1}{1+a_{1} q^{-1}+\cdots+a_{l} q^{--}} v(k)
\end{aligned}
$$

or, with $\bar{b}_{j}=\alpha b_{j} \quad j=0,1, \ldots, n$,

$$
\begin{aligned}
y(k) & =\bar{b}_{0} u(k-d)+\cdots+\bar{b}_{n} u(k-(n+d)) \\
& -a_{1} y(k-1)-\cdots-a_{l} y(k-l)+v(k)
\end{aligned}
$$

Define the parameter vector of the linear part as

$$
\theta_{l}=\left[\bar{b}_{0}, \ldots, \bar{b}_{n}, a_{1}, \ldots, a_{l}\right]^{T}
$$

where from (19) we have that $k$ must be larger than both $n+d$ and $l$ and, without loss of generality, we assume $l \geq n+d$ so that $k$ starts from $l+1$. Also introduce

$$
Y=[y(l+1) y(l+2) \cdots y(N)]^{T}
$$




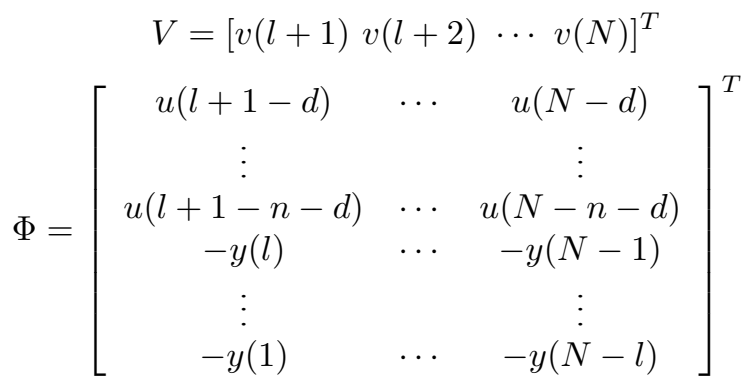

then (19) can be rewritten as

$$
Y=\Phi \theta_{l}+V
$$

Hence the first estimate, $\hat{\theta}_{l}^{1}$, can be obtained using the least squares method as

$$
\left[\hat{\bar{b}}_{0}^{1}, \ldots, \hat{\bar{b}}_{n}^{1}, \hat{a}_{1}^{1}, \ldots, \hat{a}_{l}^{1}\right]^{T}=\hat{\theta}_{l}^{1}=\left(\Phi^{T} \Phi\right)^{-1} \Phi^{T} Y
$$

Using (9), the gains of $f(u)$ and $G(q)$ are not unique. In order for these to be uniquely identifiable, we normalize the gains, e.g., set $\hat{b}_{0}^{1}=1$. If $\hat{b}_{0}^{1}=0$, set the first non-zero $\hat{\bar{b}}_{j}^{1}$ to be equal to one.

\section{B. Two-stage Identification method: Triangular ramp test}

In order to identify the nonlinear part, a triangular ramp test is applied to muscle, which is rich enough to excite the whole nonlinearity. Fig. 4 shows a typical muscle response to a triangular ramp input.

1) Stage One: Identify the nonlinear part using the linear parameters $\hat{\theta}_{l}^{1}$ estimated in the preliminary study. Without loss of generality, we assume $\hat{\bar{b}}_{0}^{1} \neq 0$ and hence the intermediate signal $w(k)$ can be obtained using

$$
\begin{aligned}
w(k) & =\hat{G}(q)^{-1} y(k) \\
& =\frac{1+\hat{a}_{1}^{1} q^{-1}+\cdots+\hat{a}_{l}^{1} q^{-l}}{q^{-d}+\frac{\hat{b}_{1}^{1}}{\hat{b}_{0}^{1}} q^{-(d+1)}+\cdots+\frac{\hat{b}_{n}^{1}}{\hat{b}_{0}^{1}} q^{-(n+d)}} y(k)
\end{aligned}
$$

The parameter vector of the nonlinear part

$$
\theta_{n}=\left[\beta_{1}, \beta_{2}, \ldots, \beta_{m+2}\right]^{T}
$$

can be estimated by fitting a cubic spline function in a least square sense

$$
\hat{\theta}_{n}=\arg \min _{\hat{\theta}_{n}} \frac{1}{N} \sum_{k=l+1}^{N}\left(w(k)-\sum_{p=1}^{n} g_{p}\left(u(k), \hat{\theta}_{n}\right)\right)^{2}
$$

2) Stage Two: Optimize the linear parameters using the nonlinear parameters $\hat{\theta}_{n}$ estimated in Stage One.

Once the nonlinear parameters are available, the intermediate signal $w(k)$ can be easily computed using

$$
w(k)=f(u(k))=\sum_{p=1}^{n} g_{p}\left(u(k), \hat{\theta}_{n}\right)
$$

This is now a general linear system identification problem and hence can identify $G(q)$ again to optimize the linear parameters to obtain $\hat{\theta}_{l}^{2}$.

This two-stage method detailed above can be repeatedly executed until convergence is achieved. Since the initial value
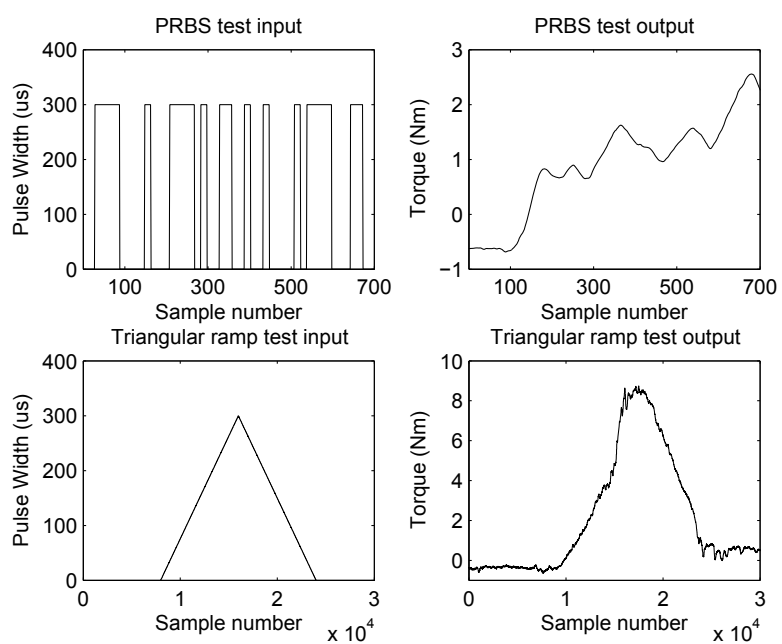

Fig. 4. Example of input and output in PRBS test and the Triangular ramp test. The stimulus frequency is $40 \mathrm{~Hz}$. The sample frequency is $1600 \mathrm{~Hz}$. In the PRBS test, the input pulse width switches between 0 and $300 \mu s$. In the Triangular ramp test, the pulse width rising from 0 to $300 \mu s$ and then back to 0 .

of linear parameters from the preliminary study is already a good estimate for the true system, we have reason to believe that just through one iteration, the optimized model can achieve acceptable accuracy with, crucially for this application area, short identification time. Next we compare the performance against two other methods that have been applied in the same general area.

\section{Two other Identification Methods}

\section{A. Ramp Deconvolution Method}

In this method, the Isometric Recruitment Curve (IRC) is estimated by deconvolving the response of a muscle to a ramp input [11] and the Linear Activation Dynamics (LAD) is represented by a critically damped second-order system [12]. This method has already been used to identify models of the arm dynamics of stroke patients in the research programme on which this paper is based and it is implemented as follows. First, a triangular input is applied with 5 seconds up and 5 seconds down and the elbow torque is recorded. Second, the elbow torque is deconvolved using the Linear Activation Dynamics (LAD). When plotted against the applied pulsewidth, this provides two isometric recruitment curves, corresponding to the increasing and decreasing ramps respectively. Then the expression

$$
f(u)=a_{1} \cdot \frac{e^{a_{2} u}-1}{e^{a_{2} u}+a_{3}}
$$

is selected to fit data in a least square sense. Third, $h(\tau)$ is convolved with $f(u)$ to produce the overall nonlinear dynamical model. The transfer function of the Linear Activation Dynamics (LAD) is

$$
h(s)=\frac{1}{T_{\omega}^{2} s^{2}+2 T_{\omega} s+1}
$$


and $T_{\omega}$ is the time constant. The natural frequency $f_{\omega}=\frac{1}{T_{\omega}}$ of the system is chosen, based on evidence in the literature cited above, as $0.85 \pi$.

\section{B. Separable Least Squares (SLS) Optimization Algorithm}

The Separable Least Squares (SLS) Optimization Algorithm [18] is another method to identify Hammerstein Structures. In this method, the linear dynamics is described by its impulse response (IRF), $h(\tau)$, which is assumed to be of finite length $T=41$. The static nonlinearity $f(u)$ is given by (30), including three nonlinear parameters $a_{1}, a_{2}$ and $a_{3}$. Thus, the model output is

$$
\hat{z}(t)=\sum_{\tau=0}^{T-1} h(\tau) f(u(t-\tau)) .
$$

and it is obvious that this is a linear function of the filter weights $h(\tau)$ and is nonlinear in the parameters $a_{1}, a_{2}$ and $a_{3}$. Thus, a parameter vector $\theta$, including filter weights and nonlinear parameters is used to represent model output as

$$
\theta=\left[h(0), \cdots, h(T-1), a_{1}, a_{2}, a_{3}\right]^{T}=\left[\theta_{l}^{T} \mid \theta_{n}^{T}\right]^{T}
$$

The SLS method performs the iterative search only for the nonlinear parameters to find the parameter vector $\theta$, that minimizes the cost function

$$
V_{N}(\theta)=\frac{1}{2 N} \sum_{t=1}^{N} \epsilon^{2}(t, \theta) .
$$

where $\epsilon(t, \theta)=z(t)-\hat{z}(t, \theta)$ and is the error between model output and measured output, and $N$ is the number of samples of the input and output signals.

\section{Simulation Results}

In order to investigate the relative performance of the three identification methods, we first apply them on data from a simulated muscle system and choose different noise levels. The simulated muscle system is defined as follows:

1) The nonlinearity $f(\cdot)$, shown in Fig. 5, is a sigmoid function:

$$
f(u)=a_{1} \cdot \frac{e^{a_{2} u}-1}{e^{a_{2} u}+a_{3}}
$$

where $a_{1}=6.8994, a_{2}=0.0410$ and $a_{3}=2.3897 \times$ $10^{3}$.

2) The linear dynamics $G(q)$ is an underdamped second order system with $T_{\omega}=0.5284, \zeta=0.6369$ and unit gain.

3) The noise levels considered are:

- $e(t)=0$ - no noise.

- $e(t)$ - normally distributed random process with zero mean and standard deviation $\lambda$ ranging over the values $0.02,0.04,0.06,0.08,0.10$.

This is the model corresponding to one of the stroke participants taking part in clinical trials, and has been used in the stimulation controller applied during their treatment. Analysis of the extensive experimental data collected throughout the intervention (consisting of upto 25 sessions of 1 hour duration) reveals it to be accurate. During tests the

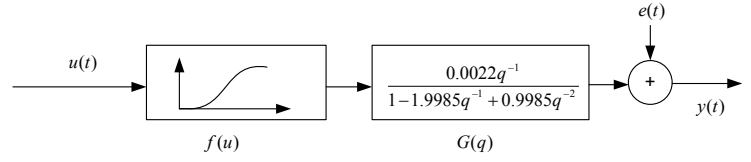

Fig. 5. Simulated Muscle System

application of FES using this model in conjunction with a simple structure ILC algorithm [3] generally led to a mean tracking error of less than $15 \mathrm{~mm}$, and in some cases less than $5 \mathrm{~mm}$. Comparison with the models corresponding to the remaining stroke participants taking part in the clinical trial also reveals that it is representative of all the participants tested. The accuracy of the model is illustrated in Fig. 6 which shows the response of the simulated muscle system in comparison with experimental data from a stroke patient's muscle. The responses can be seen to be in close agreement.

The model will therefore be adopted as representative of the muscle of stroke patients, and will be used to compare the efficacy of the identification methods that have been described. For each noise level, 100 independent trials have been performed and the mean and standard deviations of the best fit rates have been calculated, see Table I. In the noise-free case, the two-stage method can be observed to almost reconstruct the simulated system, although the variance is larger than Ramp Deconvolution and SLS, it is still comparably small enough not to significantly influence performance. It can be seen from Fig. 7 that under different noise levels, the three methods degrade but the method developed here is superior to the other two.

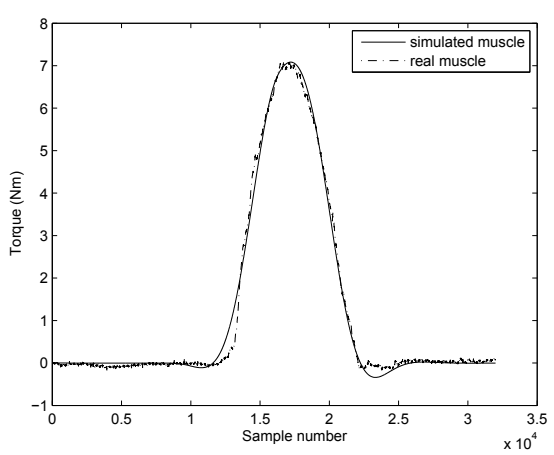

Fig. 6. Comparison of the responses of the simulated muscle system and the real muscle of a stroke patient to a triangular ramp test

\section{CONCLUSIONS AND FUTURE WORK}

In this paper a two-stage identification method was developed to identify the nonlinear dynamics of electrically stimulated muscle under isometric conditions. This method needs a preliminary experiment comprising a PRBS test and then a triangular ramp test before treatment. The proposed method is shown to outperform the Ramp Deconvolution method and Separable Least Square method on a simulated muscle system with different noise levels. 
TABLE I

BEST FIT (\%) FOR DIFFERENT NOISE LEVELS

\begin{tabular}{|c|c|c|c|}
\hline \hline & Two-Stage & Ramp Deconv & SLS \\
\hline noise-free & 99.98 & 91.24 & 92.06 \\
\hline$\lambda=0.02$ & $99.07 \pm 0.07$ & $91.19 \pm 0.00$ & $92.01 \pm 0.01$ \\
$\lambda=0.04$ & $98.14 \pm 0.02$ & $91.07 \pm 0.00$ & $91.87 \pm 0.00$ \\
$\lambda=0.06$ & $97.27 \pm 0.03$ & $90.86 \pm 0.00$ & $91.64 \pm 0.00$ \\
$\lambda=0.08$ & $96.31 \pm 0.15$ & $90.58 \pm 0.00$ & $91.32 \pm 0.01$ \\
$\lambda=0.10$ & $95.33 \pm 0.22$ & $90.24 \pm 0.00$ & $90.94 \pm 0.00$ \\
\hline
\end{tabular}

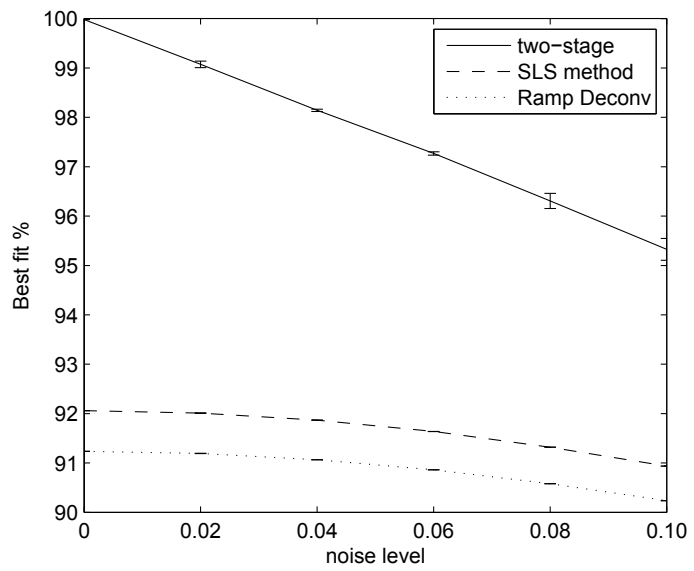

Fig. 7. Simulation results for different noise levels

Future work includes experiments on the stroke patients. The workstation and its peripheral systems for the experiments are given in [4]. Experiments will enroll more stroke patients and tests and trials will be carried out on them. All these methods will be applied on the experimental data to determine the most promising method, balancing between efficiency and accuracy.

Future work also includes extension to non-isometric condition where Passive Torque-Angle and Torque-Velocity relation $h(x, \dot{x})$ and Active Torque-Angle and Torque-Velocity relation $g(x, \dot{x})$, in Fig. 2, will be introduced. Separate experiments will be designed and an identification procedure will be implemented in line with [10].

\section{REFERENCES}

[1] J. Mant, D. Wade, and S. Winner, "Health care needs assessment:stroke", Health care needs assessment: the epidemiologically based needs assessment reviews, A. Stevens, J. Raftery, J. Mant and S. Simpson, Eds. Oxford: Radcliffe Medical Press, 2004.

[2] C. Gowland, H. deBruin, J. V. Basmajian, N. Plews, and I. Burcea, "Agonist and Antagonist Activity During Voluntary Upper-Limb Movement in Patients with Stroke", Physical Therapy, vol. 72, no. 9, September 1992, pp 624-633.

[3] C. T. Freeman, A. M. Hughes, J. H. Burridge, P. H. Chappell, P. L. Lewin, and E. Rogers, "A Model of the Upper Extremity Using FES for Stroke Rehabilitation", ASME Journal of Biomechanical Engineering, vol.131, March 2009, 031011-1 - 031011-12.

[4] C. T. Freeman, A. M. Hughes, J. H. Burridge, P. H. Chappell, P. L. Lewin, and E. Rogers, "An experimental facility using functional electrical stimulation for stroke rehabilitation of the upper limb", inProceedings of 10th IEEE International Conference on Rehabili- tation Robotics, Noordwijk aan Zee, The Netherlands, June 2007, pp. 393-400.

[5] S. J. Dorgan and M. J. OMalley, "A Nonlinear Mathematical Model of Electrically Stimulated Skeletal Muscle", IEEE Transactions on Rehabilitation Engineering, vol. 5, no. 2, June 1997, pp 179-194.

[6] F. Previdi, "Identification of black-box nonlinear models for lower limb movement control using functional electrical stimulation", Control Engineering Practice, vol. 10, no. 2, January 2002, pp 91-99.

[7] L. Bernotas, P. E. Crago, and H. J. Chizeck, "A Discrete-Time Model of Electrically Stimulated Muscle", IEEE Transactions on Biomedical Engineering, vol. 33, no. 9, September 1986, pp 829-838.

[8] G. Shue, P. E. Crago and H. J. Chizeck, "Muscle-Joint Models Incorporating Activation Dynamics, Moment-Angle, and MomentVelocity Properties", IEEE Transactions on Biomedical Engineering, vol. 42, no. 2, February 1995, pp 212-223.

[9] H. J. Chizeck, S. Chang and R. B. Stein, "Identification of Electrically Stimulated Quadriceps Muscles in Paraplegic Subjects", IEEE Transactions on Biomedical Engineering, vol. 46, no. 1, January 1999, pp 51-61.

[10] W. K. Durfee and K. I. Palmer, "Estimation of Force-Activation, Force-Length, and Force-Velocity Properties in Isolated, Electrically Stimulated Muscle", IEEE Transactions on Biomedical Engineering, vol. 41, no. 3, March 1994, pp 205-216.

[11] W. K. Durfee and K. E. MacLean, "Methods for Estimating Isometric Recruitment Curves of Electrically Stimulated Muscle", IEEE Transactions on Biomedical Engineering, vol. 36, no. 7, July 1989, pp 654667.

[12] R. Baratta and M. Solomonow, "The Dynamic Response Model of Nine Different Skeletal Muscles", IEEE Transactions on Biomedical Engineering, vol. 37, no. 3, March 1990, pp 243-251.

[13] W. Farahat and H. Herr, "A Method for Identification of Electrically Stimulated Muscle", Proceedings of the 2005 IEEE Engineering in Medicine and Biology 27th Annual Conference, Shanghai, China, September 1-4, 2005.

[14] T. Schauer, N. -O. Negard, F. Previdi, K. J. Hunt, M. H. Fraser, E. Ferchland, and J. Raisch, "Online identification and nonlinear control of the electrically stimulated quadriceps muscle", Control Engineering Practice, vol. 13, no. 9, September 2005, pp 1207-1219.

[15] P. H. Mccrea, J. J. Eng, and A. J. Hodgson, "Time and Magnitude of Torque Generation is Imparied in Both Arms Following Stroke", Muscle Nerve, vol. 28, 2003, pp 46-53.

[16] K. J. Hunt, M. Munih, N. N. Donaldson, and F. M. D. Barr, "Investigation of the Hammerstein Hypothesis in the Modeling of Electrically Stimulated Muscle", IEEE Transactions on Biomedical Engineering, vol. 45, no. 8, August 1998, pp 998-1009.

[17] M. Munih, K. J. Hunt, and N. N. Donaldson, "Variation of recruitment nonlinearity and dynamic response of ankle plantarflexoes", Medical Engineering and Physics, vol. 22, no. 2, 2000, pp 97-107.

[18] D. T. Westwick and R. E. Kearney, "Separable Least Squares Identification of Nonlinear Hammerstein Models: Application to Stretch Reflex Dynamics", Annals of Biomedical Engineering, vol. 29, no. 8, August 2001, pp 707-718.

[19] L. Ljung, System Identification: Theory for the User, 2nd ed. Englewood Cliffs, NJ: Prentice Hall, 1999.

[20] C. De Boor, A Practical Guide to Splines, ser. Applied Mathematical Sciences. New York: Springer-Verlag, 1978, vol. 27. 